\title{
L'hospitalité des humains envers les jnûn
}

Micro-scènes rituelles et écosophiques chez les Jbala du nord du Maroc

The hospitality of humans towards jnûn: Ritual and ecosophical micro-scenes among the Jbala of Northern Morocco

\section{Araceli González Vázquez}

\section{OpenEdition}

\section{Journals}

\section{Édition électronique}

URL : https://journals.openedition.org/ethnoecologie/3097

DOI : 10.4000/ethnoecologie.3097

ISSN : 2267-2419

\section{Éditeur}

Laboratoire Éco-anthropologie

\section{Référence électronique}

Araceli González Vázquez, "L'hospitalité des humains envers les jnûn », Revue d'ethnoécologie [En ligne], Supplément 1 | 2017, mis en ligne le 17 octobre 2017, consulté le 19 novembre 2021. URL http://journals.openedition.org/ethnoecologie/3097 ; DOI : https://doi.org/10.4000/ethnoecologie. 3097

Ce document a été généré automatiquement le 19 novembre 2021.

\section{c)}

Revue d'ethnoécologie est mis à disposition selon les termes de la licence Creative Commons Attribution - Pas d'Utilisation Commerciale - Pas de Modification 4.0 International. 


\title{
L'hospitalité des humains envers les jnûn
}

\author{
Micro-scènes rituelles et écosophiques chez les Jbala du nord du Maroc \\ The hospitality of humans towards jnûn: Ritual and ecosophical micro-scenes \\ among the Jbala of Northern Morocco
}

Araceli González Vázquez

1 Différents travaux ethnographiques et anthropologiques, publiés dans les deux dernières décennies, ont contribué à «repeupler les Sciences Sociales » (Houdart \& Thiery 2011), particulièrement en faisant une nouvelle place aux non-humains, mais aussi aux objets/aux choses/aux artefacts. Au sein et autour de ce qu'on appelle le «tournant ontologique» de l'Anthropologie contemporaine (Henare, Holbraad \& Wastell 2007, Holbraad \& Pedersen 2017), plusieurs ethnologues et anthropologues se sont intéressés, parmi d'autres, au pluralisme ontologique et aux cosmologies, aux relations interspécifiques -par exemple, aux rapports humains/animaux non-humains (Descola 2005, Viveiros de Castro 1998, 2002 et 2009, Kohn 2007, Kirksey \& Helmreich 2010). Ce que l'on appelle le «tournant ontologique» est étroitement lié à quelques autres « tournants » et « retours » (turns et returns, comme exprimé par Da Col 2014) de l'Anthropologie du présent, comme le «tournant animaliste» et le «tournant matérialiste ». Les non-humains (parmi eux, les animaux) et les matériaux (ou bien, la matérialité) ont été réintégrés dans le social, et ont été resitués dans plusieurs études ethnographiques et anthropologiques publiées au cours des dernières années. Dans les recherches menées au nord de l'Afrique, ces réintégrations et ces ré-situations ont été plus faibles, malgré, par exemple, l'importance accordée aux non-humains, particulièrement aux jnûn, dans les sociétés à majorité musulmane.

2 L'hospitalité, plus particulièrement son caractère normatif, est un des thèmes classiques de l'anthropologie sociale, souvent traité à propos des sociétés méditerranéennes (Pitt-Rivers 1968 et 1977, Herzfeld 1980 et 1987). En dépit d'un regain d'intérêt récent de l'anthropologie sociale pour le thème de l'hospitalité (Candea \& Da Col 2012), l'imaginaire et la pratique de l'hospitalité interspécifique demeurent 
peu explorés, notamment pour ce qu'ils nous disent, par exemple, sur les conceptions de l'altérité, de l'humanité et de l'animalité, sur les ontologies locales, sur le raisonnement éthique, sur l'activité rituelle des femmes et des hommes, et sur les relations entre les êtres vivants, en particulier sur les relations entre les êtres humains et les êtres non-humains. Malgré quelques ouvrages récents, surtout des articles, peu de travaux ont en effet cherché à entreprendre une exploration systématique des discours et pratiques associées à l'hospitalité des humains envers d'autres êtres vivants ${ }^{1}$. Mais, dernièrement, plusieurs anthropologues ont mené des enquêtes sur la manière dont les actions humaines sur la matière se déploient dans le cadre, toujours particulier et complexe, des sociabilités interspécifiques. Notre article s'inscrit dans cette ligne de réflexion sur l'Autre, et s'attache particulièrement à explorer les enjeux de l'hospitalité interspécifique au nord du Maroc.

Certes les travaux de quelques anthropologues ont mis en lumière la façon dont les usages sociaux liés à la réception de l'Autre constituent un ordre juridique, ou quasijuridique. À cet égard, on parle très souvent des « lois » (Morgan 1881, Pitt Rivers 1977), des « règles » et des «normes» de l'hospitalité, des «codes» et des "principes» de l'hospitalité, et des «éthiques» de l'hospitalité (Innerarity 2009). Et moins fréquemment, on parle de la "pensée » (Mallet 2008, à propos de Derrida) ou du «sens» de l'hospitalité (Gotman 2001). Mais, quel statut accorder aux usages de l'hospitalité envers les Autres non-humains/autres qu'humains?

4 Cet article vise à retracer quelques recherches ethnographiques et anthropologiques sur les jnûn que j'ai menées depuis l'année 2000 au nord du Maroc dans la qabila ${ }^{2}$ Ghzawa, dans les provinces de Chefchaouen et Ouezzane, dans les villes de Chefchaouen et Ouezzane, et dans d'autres qabila-s Jbala comme Al-Akhmas, Beni Mestara et Beni Mesguilda. Depuis 2014, et dans le cadre d'un Projet International de Coopération Scientifique (PICS) France-Maroc (CNRS-CNRST) intitulé " La montagne et ses savoirs ", j'ai mené aussi deux terrains en collaboration: le premier, en février 2014, avec la dialectologue Montserrat Benítez Fernández, chez les Jbala à Ouezzane, et le deuxième, en mars 2014, avec les dialectologues Ángeles Vicente et Amina Naciri Azzouz, chez les Ghomara des côtes de la Méditerranée, dans les qabila-s de Beni Zyat et de Beni Bouzra, et les Jbala des qabila-s d'Anjra et Haouz. Pour autant, les données ethnographiques ont été recueillies dans différents endroits du Rif occidental, et dans différents moments des dernières deux décennies.

Dans cet article, je suggère, principalement à partir de matériaux ethnographiques recueillis chez les Jbala du nord du Maroc, tout au long de mes travaux de doctorat et post-doctorat, depuis 2000, mais aussi à travers des exemples qui proviennent de la bibliographie ethnologique sur le Maroc, qu'une analyse plus approfondie de la productivité rituelle et symbolique au regard des jnûn permettrait de mieux comprendre les dispositifs de reconnaissance d'agentivité non-humaine, et l'établissement des systèmes de valeurs intersubjectives, liés aux éthiques environnementales. Les Jbala dont je parle habitent des villages de montagne dans le Rif occidental. Ils sont agriculteurs et pasteurs. Les paysans jbala, et la plupart des Ghomara, sont des arabophones depuis longtemps, et ils sont aussi des musulmans sunnites.

6 Dans le cadre restreint de cet article, plusieurs cas concrets sont présentés. À partir de quelques micro-scènes rituelles, on pourrait interroger et éclairer les spécificités de quelques usages actuels de l'imaginaire de l'hospitalité pour énoncer des discours sur 
les relations individuelles entre les humains et les jnûn. Au nord du Maroc, chez les Jbala, les jnûn sont conçus comme des êtres individués, avec des corps invisibles pour les humains, capables de pénétrer à l'intérieur des corps humains et animaux, notamment d'habiter ou cohabiter avec les humains et les animaux à l'intérieur de leurs corps, et capables de prendre le pouvoir et l'exercer sur l'humain et sur l'animal. C'est l'état décrit par les interlocuteurs à travers les mots du marocain mejnūn (terme générique), meskūn (habité), medrūb (frappé) ou memlūk (possédé). Mais aussi par d'autres mots qui font référence au domaine de la parenté : on dit mariée avec un jinn ou marié avec une jinnija pour parler des humains mariés avec les jnûn (pl. al-insan mjuwwjin maS l-jnûn) (González Vázquez 2013). Normalement, cet état est attribué aux « possédés » de longue durée, ceux qui sont " parasités » par les jnûn. Nous n'avons pas d'espace ici pour approfondir cette notion, mais nous avons déjà traité cette question dans notre article publié en 2013. Tel « mariage ", traité souvent dans les livres de figh musulmanes, transforme les jnûn en affins, en parents par alliance des humains. Mais, est-ce que l'affinisation d'Autrui est aussi une pratique activée avec tous les hôtes ?

Dans son œuvre «Les trois écologies » (1989), le philosophe Félix Guattari (1930-1992) développe la notion d'« écosophie » en tant qu'articulation éthico-politique et éthicophilosophique complexe entre trois registres écologiques, «celui de l'environnement, celui des rapports sociaux et celui de la subjectivité humaine » (Guattari 1989, Antonioli 2014). Avant Guattari, Arne Naess, philosophe norvégien, forge le même concept d'écosophie pour souligner que l'homme s'inscrit dans l'écosphère comme une partie de tout le vivant, et pour proposer une philosophie de l'environnement et une éthique environnementale (Naess 1973 : 100). Dans cet article, la fertilité analytique du concept d'écosophie, qui dans son sens primordial étymologique, nous parle des savoirs sur les environnements, et sur les savoirs dans les relations interspécifiques, nous permettra de réfléchir sur un sujet partiellement esquissé dans l'anthropologie des altérités que j'ai développé dans ma thèse de doctorat, intitulée Femmes de Jbala: Genre, Islam et altérités au Maroc, et soutenue à l'Université de Cantabria (nord de l'Espagne) en 2010. Plus précisément, je me propose de présenter ici certaines praxis écosophiques, liées aux micropolitiques de l'être, de l'être-avec et de l'être-ensemble au Rif occidental, et qui doivent être comprises aussi en connexion avec l'élaboration et la transmission des savoirs sur l'Autre. Je me propose aussi d'exprimer dans quelle mesure l'hospitalité est une pratique rituelle, mais aussi écosophique, révélatrice des connaissances et des savoirs sur l'Autre et sur les repositionnements des êtres dans leur environnement.

C'est dans les petits hameaux de qabila Ghzawa, enclavés dans les vallées profondes des montagnes du Rif occidental, de la région des Jbala, au nord du Maroc, que j'ai commencé à m'interroger sur les diverses manières dont les femmes vivent, perçoivent et définissent leur engagement avec les Autres, humains et non-humains, et sur la pluralité des rapports entre les existants, particulièrement entre les femmes et les hommes, les femmes et les jnûn, mais aussi entre les femmes et les saints, les femmes et les morts, et les femmes et les dormants ${ }^{3}$. Je voudrais présenter ici quelques réflexions sur un sujet d'étude dont les limites ne sont pas toujours nettes. Il s'agit de rendre explicite ce que quelques paysannes Jbala des hameaux montagnards expriment sur la façon dont elles conçoivent l'hospitalité envers les non-humains, et plus particulièrement, le don du couscous aux non-humains. 


\section{Les enjeux de l'hospitalité}

9 Immanuel Kant, le philosophe allemand, conçoit que tous les hommes, les humains, ont droit à l'hospitalité (Kant 1795). L'hospitalité, chez Kant, a un caractère limité et paradoxal (Shérer 1997) : elle doit être universelle, mais les non-humains restent exclus de cette universalité. L'universalité kantienne concerne un univers humain. L'importance accordée au vivant non-humain aujourd'hui, à certains d'entre eux, notamment aux animaux non-humains et aux végétaux, amène à penser les limites de l'hospitalité universelle humaine kantienne. Hélène Cixous souligne ces limites. Elle écrit : « Mais, le non-humain, l'animal par exemple, est exclu. Soit. Pas de chat chez moi alors? Et les dieux?» (Cixous 2003). Cixous ne fait que reprendre ici les mots du philosophe français Jacques Derrida. Il écrit : «Si l'on ne fait pas droit à l'hospitalité envers l'animal, c'est aussi le dieu que l'on exclut » (Derrida \& Dufourmantelle 1997 : 126). Derrida parlait aussi d'une hospitalité envers le « ver à soie » (Derrida $1998: 82-4$ ). Ce faisant, il montrait l'intérêt qu'il portait à la question de la frontière entre l'animal et l'humain. Ici, nous voulons reprendre un peu l'esprit de ces réflexions derridiennes en explorant la frontière ontologique des humains, des animaux et des jnûn, tout en prenant en compte la façon dont l'hospitalité, comme souligné par Roy Wagner, « takes the form of social boundary maintenance » (2012: S162), définie par l'auteur comme " the scrupulous cozening of the stranger or outsider in what Pitt-Rivers called the Mediterranean defence', ou 'defence in depth'» (2012:S162).

Quand on parle de «lois ", de "règles » et de "normes » d'hospitalité au Maroc et au nord de l'Afrique, on présuppose généralement la participation d'agents humains. En fait, c'est l'hospitalité entre humains qui a fait l'objet des réflexions les plus nombreuses, souvent sur le thème de l'hospitalité "arabe », "berbère » et "marocaine» (Camps-Fabrer 2000, Skounti 2006). De façon similaire à ce qui a été constaté par d'autres études (Candea 2012), il y a tout un discours très répandu sur les « traditions » et la « culture » de l'hospitalité au Maroc. L'hospitalité est souvent perçue comme une sorte de vertu nationale, ou comme une partie essentielle de l'identité, de l'être " marocain ", " arabe » ou " berbère ». Un article d'Ahmed Skounti a traité aussi la question de l'hospitalité et de la sainteté, ce qu'il appelle, d'après Louis Massignon (1987), «l'hospitalité sacrée». Mais, est-ce qu'il existe une hospitalité des humains envers les non-humains? Tel que signalé par Skounti, l'hospitalité des humains envers les humains est parfois régie par des prescriptions d'ordre juridique (2006: 150). L 'hospitalité des humains envers les non-humains, est-elle structurée par les mêmes « ordres juridiques » que celle qui concerne seulement les humains?

11 On connaît l'importance des repas d'hospitalité dans tout le nord de l'Afrique. À l'exception de quelques études récentes (Rhani 2007 et 2008, Gélard 2008, González Vázquez 2010), et de quelques notes peu nombreuses dans les travaux, dont ceux de Westermarck (1926), Crapanzano (1973), Rachik (1990), Garrigues-Cresswell (1994), Mahdi (1999), Hell (2002), Rhani (2007 et 2008) et Gélard (2008), la productivité symbolique des rites d'hospitalité des humains envers les jnûn au Maroc demeure peu explorée.

12 Ritual and belief in Morocco, publié par Edward Westermarck à Londres en 1926, nous offre quelques exemples des « rituels d'hospitalité » des humains envers les jnûn. Ces exemples concernent des groupes arabophones et berbérophones du Maroc. Nous aurons l'occasion de revenir sur l'un de ces exemples, mais il ne serait pas inintéressant 
d'avancer maintenant que Westermarck a écrit en détail sur la diyāfa (arabe, « repas d'hospitalité »), et qu'il a lié cette pratique avec l'Yār (traduit en anglais par « shame », et défini comme « transference of a conditional curse ») et la șadāqa (don, offrande).

Vincent Crapanzano (1973 : 159) parle de la nourriture offerte aux jnûn. Il se réfère aux «exorcistic cures» et "sacrifices»: "(practices) they may involve the ritualized preparation of special food such as a chicken cooked without salt (messūs), which is fed to the patient (that is, to the jinn with him) ». Crapanzano ajoute : « both sacrifices and the eating of special foods (diyāfa) are not restricted to exorcistic cures; they are often part of the symbolic cures as well ».

Les rituels d'isgar des berbérophones du Haut-Atlas et de l'Anti-Atlas soulignent les relations des humains et des non-humains. Ce sont des rituels sacrificiels, d'alliance avec les jnûn. L'ouvrage Sacré et sacrifice dans le Haut-Atlas marocain, publié par Hassan Rachik, offre une information détaillée sur l'isgar et les nourritures pour les jnûn dans le Haut-Atlas (1990 : 58). Il s'agit d'un rituel non sanglant, parfois considéré comme une expression sacrificielle, liant les femmes au monde des jnûn. Rachik souligne que l'homme est associé " au sacrifice sanglant orthodoxe et aux divinités bienfaisantes ", et la femme "au don alimentaire hétérodoxe et aux jinn" (1990: 107). Parlant des berbérophones, les Idaw Martini de l'Anti-Atlas, Narjys El Alaoui (2001) identifie aussi l'isgwar. Dans le Haut-Atlas berbérophone, Garrigues-Cresswell (1994:184) a défini l'isgwar de cette façon:

« il est nécessaire qu'avant l'immolation des animaux soit effectué l'isgwar. Il s'agit de débarrasser un lieu des puissances dangereuses voire malveillantes, "les autres ", «ceux que l'on ne voit pas ", "ceux que l'on ne peut nommer», car le terme de jnun doit être absolument évité. Ce rite, qui dans la division sexuelle des tâches rituelles incombe aux femmes, consiste à délayer un peu de farine avec de l'eau, sans y adjoindre de sel et à déposer un peu de cette préparation en silence et en utilisant la main gauche pour délimiter un espace «sacré » en calmant les jnun par cette offrande d'une nourriture appropriée, non salée - contrairement à la nourriture destinée à l'alimentation humaine qui doit être salée - pour qu'ils reçoivent leur part et s'éloignent ».

Dans sa monographie sur les Gnawa, Hell (2002 : 185), en parlant des jnûn, exprime que « un morceau de viande sacrificielle, cuite sans sel, lui est donné à manger ». Hell parle de "nourriture votive" destinée aux jnûn, une nourriture qui "doit toujours être préparée sans sel » (Hell 2002 : 209). La présence de nourritures sans sel dans les rituels des Gnawa est très bien attestée ainsi que les sacrifices sanglants pour les jnûn (Kapchan 2007 : 75).

16 Tout en se référant à l'ambivalence de la notion d'hospitalité en latin (où hostis désigne à la foi l'hôte et l'ennemi), Rhani (2007 et 2008) constate l'existence de gestes hostiles et de gestes d'hospitalité au sein d'un même rituel, opérés par une femme qu'il présente comme "possédée-thérapeute nourricière", prenant ici en compte le concept derridien de "l'hospitalité »4. En parlant de cette pratique rituelle, aussi dans le mausolée de Ben Yeffu, Maarouf (2009) parle d'« evil expulsion sacrifice », et Gélard, en parlant de la nourriture sans sel au sud du Maroc, de "nourriture non-humaine », de «nourriture démoniaque », et de "repas démoniaque» (2008: 96). Nina Consuelo Epton, dans son livre Saints and sorcerers: A Moroccan journey, définit la « diffa » comme une sorte de " peace-offering to jinns» $(1958: 197)$.

Au-delà de la démarche spécifique illustrée par Crapanzano, Hell et Rhani, parmi d'autres, qu'est celle de la démarche thérapeutique, nous voulons consacrer notre 
réflexion aux formes multiples de relation entre les humains et les jnûn. Plus précisément nous réfléchirons ici sur les relations de domination, de résistance, d'alliance et d'affinité, et sur l'inversion rituelle des éléments normatifs des pratiques. Ainsi, nous examinerons certaines pratiques rituelles, comme la diyā $f a$ ( «hospitalité »), la șadāqa ("don», "offrande», " aumône») et la dbịha ("sacrifice»), que nous considérons comme étant étroitement liées aux logiques d'inclusion et d'exclusion des jnûn, et au brouillage des frontières ontologiques (Descola 2005). Nous rompons aussi avec la vieille image de l'anthropologie méditerranéenne qui nous montre des hommes qui sont les hôtes d'autres hommes (men hosting men, Candea \& Da Col 2012 : S8).

De nombreuses études menées en anthropologie au Maroc ont visé à explorer la commensalité sacrificielle. Logiquement, ils dépassent le contexte rituel de l'hospitalité. Comme Gélard (2010: 75) l'indique, ils engendrent des questionnements complexes comme celui lié à la protection, aux hiérarchies, au clientélisme, aux relations d'intercession, etc. Il y a une sorte de commensalité rituelle centrée sur le couscous peu explorée, celui du tukal, qui est une sorte d'empoisonnement. En fait, ce nom est donné à la nourriture à laquelle on ajoute des ingrédients dangereux, et souvent, vénéneux. Très souvent, des matières animales. Il s'agit d'une commensalité rituelle qui concerne les humains, les animaux et les jnûn. Le poison est un élément central dans plusieurs récits hagiographiques que j'ai compilés dans le Rif occidental. Le poison est la substance qui permet aux saints (awliya) de s'approprier de la baraka d'autres saints. Ces substances toxiques font souvent partie d'un couscous que le saint hôte offre à son invité saint. Dans ces récits, une fois que l'invité saint retire le poison du couscous, il ressort de celui-ci sous la forme d'un serpent, donc d'un animal. Comme l'a noté Bloch (2005), le poison est un élément tropique qui évoque les risques de l'accueil, et les risques des substances en partage. Les saints se défendent contre les saints, et ils montrent de l'agressivité envers leurs hôtes. Lens (1925, cité dans Amster 2013 : 161) nous parle aussi de cette agressivité envers l'hôte, cette fois concentrée sur les jnûn: les « sorciers » humains traitent de la " possession par les jnûn » entraînant la victime humaine à manger un mélange qui est appelé, comme dans le cas du repas d'hospitalité, țąām («nourriture »): il s'agit d'un couscous roulé à la main morte, appelé ftel-el-miet, mélangé avec les os des morts et d'autres éléments.

Derrida nous rappelle que "nous sommes irréductiblement exposés à la venue de l'autre» (Derrida \& Dufourmantelle 1997). À l'égard d'un visiteur, nous avons deux attitudes possibles: le recevoir en fonction des règles et des usages de chez nous; et nous exposer à ce visiteur dont les règles et usages liés à sa venue sont dictés par luimême. Quelle attitude prennent idéalement mes interlocuteurs jbala face à la « visite » d'un jinn ou d'une jinnija chez soi ? Est-ce qu'il y a des règles et des usages imposés par les jnûn eux-mêmes? Dans le discours d'une femme du douar d'Ajibesh, dans la qabila d'Anjra, près de la ville de Tétouan, nous trouvons la venue de l'Autre dans l'animal. Les gens disent que les animaux voient les jnûn, et c'est pour cela que lorsqu'un jinn arrive près d'elle, une vache ne marche pas, elle lance des ruades mais elle ne s'enfuit pas. Aussi, dans son discours, le statut d'animaux (animalité) de certains animaux est mis en doute. La venue d'un jinn chez lui contribue au brouillage des frontières ontologiques. Si l'on prend les propositions de travail de Descola (2005) sur les modes d'existence et les ontologies, et si l'on examine la " physicalité » et « l'intériorité » des humains et des jnûn, on verra que, chez les Jbala et les Ghomara, elles ne sont pas appréhendées comme radicalement distinctes des humains, parce que l'intériorité des jnûn, comme l'a souligné Simenel (2012:14) dans le cas des Aït Ba Aamran, est une 
intériorité en tout point anthropomorphe. Pour autant, les jnûn sont censés être capables de traverser certaines frontières ontologiques, particulièrement, d'aller audelà de la physicalité jinn et de la physicalité humaine et aussi de la physicalité animale. Les corps des jnûn sont invisibles pour les humains (personne ne peut savoir à quoi ils ressemblent), mais les corps humains se constituent en « corps perméables». Les corps des animaux sont aussi des corps perméables, particulièrement, les corps des animaux domestiques : chiens, chats, vaches, chèvres, ânes, même si d'autres animaux sauvages le sont également bien que plus rarement.

\section{Micro-scènes rituelles et écosophiques marocaines}

La première micro-scène rituelle se déroule dans une maison rurale d'un petit village de montagne des Ghzawa. Il s'agit d'une scène du passé récent, évoquée par un homme marié d'âge moyen. Nous sommes assis ensemble, les nuques et les épaules appuyées contre le mur de la maison, et la femme de cet homme dort par terre. Je lui demande de me parler sur les jnûn, et il me parle d'un jinn qui avait habité sa femme. Ce jinn était l'hôte de sa femme $(d y \bar{a} f)$. Un fqih d'un village proche lui avait donné quelques instructions afin de faire sortir le jinn du corps de sa femme. Il a suivi les instructions. Il a placé un fil rouge autour du col de sa femme. Le jinn à l'intérieur de son corps refusait de lui permettre de le placer, mais finalement, il avait réussi à le mettre. Le fqih a indiqué qu'il devait donner l'hospitalité au jinn. Il a organisé une diyāfa, un repas rituel d'hospitalité, avec toute la famille réunie et assise à table. L'homme a sacrifié un poulet noir, et il a préparé un couscous sans sel, et un tajin de poulet. Le jinn s'est mis à manger, et immédiatement, il a commencé à se plaindre. Il n'a pas aimé le repas. L'homme et le jinn discutent, et l'homme demande au jinn de quitter le corps de sa femme. Après avoir mangé, le jinn quitte la femme et la chambre.

La deuxième micro-scène rituelle a été évoquée par un autre interlocuteur Ghzawa. Lorsque nous parlions des cimetières, il m'a raconté que les gens font des choses «interdites» (harām) dans les cimetières du village. Il s'agissait d'étrangers (barrāniyyīn), venus pour faire des sacrifices sanglants sur les pierres des sépultures de leurs ancêtres. Il m'a dit que cette dbịha est, en fait, une șadāqa, un don pour les jnûn, parce que les jnûn aiment le sang et ils peuvent donner beaucoup de pouvoir aux hommes. Il m'a raconté aussi que les gens de la fraction Bou Hassan visitent un saint local connu comme maître des jnûn, et qu'ils partagent un couscous dans le mausolée du saint. Ils le mangent avec une grande cuillère de métal (mağrüfa). Le métal est utilisé afin de mettre en fuite les jnûn, alors que la sadāqa du couscous a pour but l'obtention de la protection du saint.

La troisième micro-scène rituelle a été évoquée par une interlocutrice Ghzawa. Rahma m'a raconté qu'elle avait été paralysée par un jinn, et le figh de son village lui avait dit qu'elle était meskūna (habitée par un jinn). Pour faire sortir le jinn, elle devait préparer un repas sans sel pour toute la famille. Personne ne devait manger de ce couscous, à l'exception d'elle-même. Le fqih prévoyait que le jinn parlerait. Et, en effet, pendant le repas, le jinn avait indiqué à Rahma où elle devait placer sa part de la sadāqa. Dans ce cas, c'était dans un lieu proche du village, un lieu-dit meskūn, habité par les jnûn.

Les quatrième et cinquième micro-scènes rituelles ont été relevées dans des sources bibliographiques. Ce ne sont pas des exemples de la société Jbala ou Ghomara, mais ils 
sont très éloquents. La quatrième micro-scène rituelle est évoquée par Westermarck (1926) :

«In the Hiáina, if a person is troubled with lé-ryäh, a black or white cock or hen is killed over his head so that the blood drops down on it. The fowl is boiled without salt, and the sick man drinks the gravy and eats the meat. The feathers only are taken to a haunted place, where the jnûn, attracted by the smell of blood, have a fight for them between themselves, with the result that the patient will be left in peace and recover from his illness. This practice is known under the name of $n$ nasra; but there is also another cure, called sadaqat j-jnûn, which consists in a dish of ta'am (seksu) with meat but without salt being put over night at a haunted place near the house. It is believed that the jnûn go away from the patient together with the ta'am, not to come back again » (Westermarck 1926, i : 338).

La cinquième micro-scène rituelle vient d'un conte inclus dans la compilation de Legey dans sa thèse (2010: 49) intitulé " Histoire de la jeune fille qui naquit d'une pomme ». En mangeant une des deux pommes données à sa femme stérile par un sorcier, un homme avait "accouché d'une fille», sortie de sa jambe. Il l'abandonna sur place, et elle grandit avec les petites gazelles. Pour savoir s'il s'agit de cette fille, il fit préparer deux plats de couscous avec du sel et l'autre sans sel, et les fit déposer près de la source où buvaient les gazelles. Il pensait : "Si cet être est une fille d'Adam [humaine], elle mangera le plat salé ; si c'est une jenniya [féminine des jnûn], elle mangera le plat sans sel, car les génies ne mangent que la cuisine non salée ». Le lendemain, il alla voir ce qu'avait mangé cet être et, quand il fut bien certain que c'était un être humain, il la poursuivit et l'attrapa (Legey $2010: 49$ ). Apparemment, il s'agit d'une sorte d'ordalie présente dans d'autres contes marocains (Berger 1992 : 203).

La sixième micro-scène vient d'un conte recueilli pendant le travail de terrain développé avec Montserrat Benítez Fernández à Ouezzane, en février 2014. Dans ce récit, une ogresse prend deux enfants comme otages : elle trait sa chienne et elle leur fait du saykuk avec des poux et du lait de sa chienne. Tandis qu'une des filles ne prenait que le début (du repas), le reste des filles mangeaient et la plus petite des filles a bien mangé. La sœur ainée pinçait la petite et lui disait : «ne mange pas, la nourriture n'est pas bonne " et la pinçait à nouveau pour l'empêcher de manger. La plus petite lui répondait : «Oh sœur! Qu'est-ce que tu as? Tu ne me laisses pas manger ! ». Les autres sœurs demandaient à la sœur ainée de laisser manger la plus petite, mais elle disait « la nourriture n'est pas bonne ».

Le ghoul, dans ce cas, la ghoula, est une " ogresse ». Et cette fois, nous sommes face à un être avec une physicalité et une intériorité assez spécifique. Il s'agit d'un être qui habite la forêt et les grottes. Il y a quelques récits qui donnent à l'ogresse un caractère anthropomorphe très accusé, mais aussi un caractère zoomorphe. Une sorte d'hybride. En ce qui concerne la nourriture, il s'agit d'un couscous assez particulier: l'appelé saykuk. La ghola donne aux enfants humains à manger des animaux qui ne sont pas purs du point du vue du figh al-tahāra, et qui ne sont pas halāl.

À partir de ces micro-scènes rituelles, nous pouvons nous poser une série de questions. Quand est-ce que les humains sont les hôtes des jnûn? Et à l'inverse ? Quel est le rôle du don, du sacrifice, et de la réciprocité dans les rites de l'hospitalité envers les jnûn? Quel est le rôle des actions humaines sur la matière? De quelle façon les humains instrumentalisent les matières devant les jnûn?

Selon l'anthropologue anglais Michael Herzfeld, l'hospitalité est « un contexte majeur pour la négociation des relations sociales, permettant d'abondantes opportunités 
d'inversions symboliques des relations de pouvoir environnantes » (2008: 99). Saskia Walentowitz souligne, à propos de la société Touareg, que l'hospitalité est « le seuil où se négocient les relations avec l'altérité » (2006). L'hypothèse qui sert de fil conducteur à notre enquête est, en effet, que certains usages du langage de l'hospitalité, et des pratiques mêmes de l'hospitalité, révèlent l'énonciation verbale et matérielle d'un lien social. La mise en scène des rituels d'hospitalité, bien qu'à travers certaines inversions rituelles, a pour but de transformer l'altérité radicale de l'Autre. C'est le cas des jnûn. Et pourtant, l'hospitalité des humains envers les jnûn constitue un aspect clé dans les relations de médiation avec un Autre potentiellement dangereux. Les relations sociales de pouvoir sont centrales, en tant qu"alliance entre l'humain et le jnûn pouvant donner un pouvoir immense à l'humain, mais l'exercice du pouvoir des jnûn sur les humains «affaiblit » l'humain. Le jinn l'empêche de parler ou prendre la parole pour lui, l'empêche de bouger, lui paralyse les extrémités, perturbe sa raison ( $($ aql), ne le laisse pas dormir, le réveille dans la nuit, lui provoque des cauchemars, lui fait pousser des cris, rire sans raison ou pleurer; cause des convulsions dans son corps...

Michael Lambek a souligné que «multiple and sometimes competing constructions of spirits can coexist in the same society» $(1996: 246)$. Nous voudrions ajouter que, en fait, il y a aussi des conceptions des esprits qui coexistent dans la même personne, en particulier en raison de la nature spéculative de la relation des humains avec les jnûn. Chez les Jbala, différents agents peuvent mettre en œuvre toute une panoplie de savoirs et des pratiques sur et/ou envers les jnûn: les paysans, les fuqāha, les saints, les shurafa... Donc, nous ne pouvons pas affirmer que ces savoirs et pratiques sont universellement conçus. Comme nous allons voir, souvent il s'agit d'une co-production de l'hôte et de l'Autre. Parfois, il y a une nécessaire collaboration du «malade » et du fqih.

\section{Lois, règles et normes de l'hospitalité au Maroc}

Chez les Jbala du nord du Maroc, le modèle d'hospitalité le plus évoqué est celui de l'hospitalité codifiée : un devoir, une obligation religieuse, un principe moral, une vertu plutôt masculine, liée à l'honneur et au pouvoir.... Ainsi, le Coran ordonne de donner l'hospitalité "au compagnon et au voyageur». On offre du thé. On égorge un animal pour l'hôte (dbihha) : soit un bouc, une chèvre, un mouton, une brebis, un coq, un poulet... L'espèce animale sacrifiée est choisie en fonction du prestige accordé à l'invité. On prépare du couscous, appelé ța'am («nourriture»), avec la viande de l'animal sacrifié. Il y a une invitation à partager l'offrande de nourriture, une offrande désignée parfois comme șadaqa (« offrande », « don », " aumône »). L'hospitalité du Prophète est aussi invoquée : elle dure trois jours. On ne peut pas rester très longtemps chez l'hôte, comme les jnûn ont l'habitude de faire. Le mot arabe dịyāfā, hospitalité, est la réception faite aux hôtes. Il désigne aussi le repas d'hospitalité. Diffa renvoie, dans la bibliographie coloniale française, à l'hospitalité comme acte diplomatique des grands seigneurs, un repas d'apparat, un repas pantagruélique. L'étymologie du mot, notamment la racine arabe, sert à signifier : «ajouter, joindre, compléter ». Quand les Jbala utilisent le mot dyaf pour désigner un hôte non-humain dans le corps d'un humain, ils ne font rien de bizarre: l'exemple métaphorique du mot "hôte» est bien connu dans plusieurs sociétés méditerranéennes. Il n'est pas étranger à la langue arabe. Également, les 
scientifiques parlent d'hôte pour désigner le corps qui reçoit un agent pathogène, parfois un parasite.

Dans quelle mesure les rites d'hospitalité envers les jnûn sont-ils des rites d'institution de l'altérité ? En réagissant face à certains aspects du comportement de l'être humain et les attribuant au jinn qui l'habite, l'humain se sert des rites afin de séparer les aspects constitutifs de l'humain et ceux du non-humain, et il choisit les fragments appartenant à la non-humanité. Il semble opposer différents fragments de la personne-même, humaine, jinn/jinnija, qu'il ne parviendrait pas à faire coexister, une sorte de jihad intérieur, comme décrit par Pandolfo (1997) ou par Aufauvre (2009) dans d'autres contextes. Les rites d'hospitalité décrits sont aussi des rites d'agrégation ou des rites d'intégration.

\section{Inversions rituelles}

Les micro-scènes rituelles que nous venons de décrire dans cet article nous situent vraiment au cœur des inversions et des renversements des "lois ", "règles " et « normes » de l'hospitalité humaine.

Quel est le sens des inversions rituelles dans les rites d'hospitalité des humains vers les jnûn? Nous pouvons identifier plusieurs inversions rituelles: a) sacrifice, dbĭha: le sacrifice est fait afin de donner à manger le sang, pas de la viande, parce que le sang frais est destiné à nourrir les jnûn, qui l'aiment bien. On croit que les jnûn se nourrissent de sang et de plumes noires. Il est nécessaire d'inverser certaines lois de l'hospitalité humaine pour satisfaire le jinn ; b) couscous sans sel pour l'invité : pas avec $\mathrm{du}$ sel, qui est le symbole de l'hospitalité humaine, mais pas avec du sel pour satisfaire le jinn, qui n'aime pas le sel; c) couscous à manger à l'extérieur de la maison de l'hôte, pas à l'intérieur ; d) cuillère en métal pour que l'invité jinn mange le couscous, car son aversion pour le métal est connue; e) pas d'énonciation de la basmala au début du repas, etc. ; f) sacrilèges dans les espaces... Pas de signe du thé, du lait, des dattes... et des autres matières de l'hospitalité humaine. Du moins, dans les micro-scènes rituelles décrites dans cet article. Une des règles de l'hospitalité est de ne pas demander à l'invité qui il est, ni d'où il vient. Pitt-Rivers a souligné cela dans le cas de l'hospitalité arabe, comme rappelé par Farès dans L'honneur chez les Arabes avant l'Islam (1932 : 95). $\mathrm{Au}$ contraire, une des premières questions du fqih au jinn-hôte est qui il est (son nom) et d'où il vient, sa foi (généralement, pour savoir s'il est musulman, juif ou chrétien (Mateo Dieste 2014), à quel moment il est arrivé dans ce corps humain, pour quelle raison... Le fqih ne se comporte pas comme un bon hôte qui accueille. Il se montre comme un transgresseur.

On mange la même nourriture que l'autre, mais pas de la même façon. Dans le cas de l'hospitalité entre humains, le repas rituel scelle l'accueil, mais dans le cas de l'hospitalité des humains envers les jnûn, le repas scelle l'accord de leur départ. On a vu aussi que les jnûn sont des hôtes qui se plaignent de la nourriture. Cela casse l'une des règles de l'hospitalité, ou les humains ne doivent jamais se plaindre de l'hospitalité reçue, et encore moins en présence de l'hôte. La pratique de l'hospitalité ajoute de l'honorabilité à l'hôte. Tout au contraire, la nourriture offerte aux jnûn reste de l'honorabilité. D'un point de vue religieux, normatif, c'est une pratique considérée comme hérétique. En fait, cette pratique peut aussi « déshonorer » l'individu, la famille, la communauté, la maison, jusqu'au quartier, au village, à la fraction et à la qabila. Ce 
sont des pratiques associées à la magie. L'hospitalité envers les humains est communiquée socialement, mais l'hospitalité envers les jnûn reste le secret de l'individu ou de la famille.

En ce qui concerne les espaces, les rituels signalent les limites spatiales de l'hospitalité. Dans la première micro-scène, le fil rouge signale une limite (el -hadd). Les Jbala utilisent des fils rouges dans quelques autres rituels: placés sur les poignets des nouveau-nés, et sur les poignets et chevilles des mariées, dans les rituels de la henna (nhār el-ḥenna). Dans les cas mentionnés, le fil rouge est placé autour d'une articulation du corps humain, en montrant les parties périphériques du corps (mains, pieds et tête). Ce sont aussi des parties du corps humain qui sont très souvent endommagées par les jnûn: ils paralysent les mains et les pieds, le raisonnement ( (aql) que les Jbala placent dans la tête... Dans la deuxième micro-scène, les visiteurs du mausolée du saint utilisent un vieille cuillère métallique pour consommer le couscous. La littérature ethnographique de l'Afrique du Nord fournit de nombreux exemples de l'aversion ressentie par les jnûn au métal. En fait, l'une des façons de se protéger de la jinnija Aisha Qandisha est de placer un couteau en métal sur le sol, entre la personne et elle. Un couteau en métal est aussi placé dans le lit du nouveau-né, pour le protéger des jnûn. La maison des Jbala, appelée dār, est un lieu de l'hospitalité avec des limites marquées rituellement. Il y a des lieux spécifiques dans l'accueil: les seuils, les portes, les fenêtres, la cour et les cloisons intérieures. En ce qui concerne les jnûn, les Jbala marquent les limites de l'accueil dans l'espace domestique : ils laissent du sel dans les coins de la maison pour délimiter un espace impénétrable, ils laissent du ketran (goudron liquide) dans ces mêmes lieux pour délimiter un espace impénétrable pour les serpents, et ils ferment les portes et fenêtres pour les humains qui transmettent le mauvais œil.

Une dernière inversion rituelle est la transformation de l'étranger hostile, hostis, en l'étranger invité, hospes, comme signalé par Rivers (1977). L'hostilité des jnûn est minimisée. Les échanges, les repas d'hospitalité offerts par l'humain aux jnûn permettent une sorte de coexistence ou convivencia pacifiée, ou, du moins, une hostilité assumée du côté de l'humain.

\section{L'animal dans l'hospitalité des humains envers les non- humains}

En Afrique du Nord, les animaux de l'hospitalité sont aussi les animaux de la commensalité et du sacrifice religieux (Benkheira 1999: 91). Mais, ils ne sont pas seulement des procureurs de nourriture carnée. Dans la deuxième micro-scène rituelle, le fqih avait suggéré de sacrifier un poulet noir. Dans d'autres cas, ce sont les jnûn qui demandent la couleur de l'animal. Dans un exemple rapporté par Rhani (2008:229), les jnûn demandent aussi l'espèce animale à sacrifier, souvent une volaille. Rhani nous dit que « le thérapeute devine l'exigence sacrificielle de l'esprit sans que celui-ci l'exprime ouvertement». Dans notre deuxième micro-scène rituelle il n'y a aucun rituel divinatoire. Il y a un code mobilisé. Les fuqaha des Jbala estiment que les jnûn préfèrent les volailles noires. En tout cas, le jinn et la jinnija sont des êtres demandants. Dans un exemple fourni par Emilio Spadola dans son livre The Calls of Islam (2013), Aisha demande du pain sans sel, et différents vêtements de certaines couleurs (2013:81). 


\section{L'affinisation d'autrui}

\section{(1)} ables. L'hospitalité des humains envers les jnûn est aussi une hospitalité régie par l'ambigüité et l'instabilité. Les jnûn sont les hôtes parfois invités à s'intégrer dans l'humain, parfois invités à quitter l'humain, plus particulièrement les corps humains. L'humain exprime souvent son désir de mieux contrôler les jnûn, mais aussi de se 
protéger d'eux et de les combattre. Les jnûn sont des formes d'altérité susceptibles d'altérer l'intériorité et la physicalité humaines.

Si l'hospitalité rituelle procède, comme l'exprime Benjamin Boudou, « d'une hiérarchie renversée œuvrant à la double tâche de rendre possible la sociabilité intergroupe tout en préservant l'intégrité intra-groupe " (2012: 271), on peut dire que l'hospitalité rituelle des humains envers les jnûn, chez les Jbala du nord du Maroc, rend possible la sociabilité de l'intériorité humain et jinn anthropomorphe à l'intérieur du corps humain, et parfois veut préserver l'intégrité de l'intériorité humain en expulsant l'intériorité jinn perçue comme un intrus/transformé en un intrus. Il permet l'entretien des frontières sociales (Wagner 2012) et des frontières ontologiques (ontological boundaries), mais aussi de les manipuler. L'hospitalité est aussi un code de pacification, de résolution du conflit endogène à l'intérieur du corps humain.

En parlant de "l'ouverture à la venue de l'autre», Jacques Derrida thématise l'hospitalité à partir du fait de "l'irruption» de l'autre chez soi. Les rituels d'hospitalité des humains envers les jnûn peuvent être aussi assimilés aux rites de contrôle de l'irruption de l'Autre. Nous retournons à ce qui a été signalé par Herzfeld : Est-ce que ces rituels permettent l'inversion symbolique des relations de pouvoir ? Estce qu'on doit parler d'inhospitalité?

Les interactions des humains avec les jnûn, toujours complexes, mais fondées sur les expériences individuelles, constituent un champ de spéculation anthropologique sur l'altérité et l'identité de la personne. Aussi, ils sont des relations asymétriques (Descola 2005).

On a vu que le type de nourriture ingéré est très important dans la définition des relations entre les humains et les non-humains chez les Jbala. On a vu que le couscous non salé est la « nourriture » des jnûn, des morts, et des saints, mais aussi la nourriture de la ghola, l'être sauvage et non-humain de la forêt. C'est à travers la commensalité rituelle et sacrificielle, à laquelle on peut reconnaître une portée éthique, surtout dans le cas de l'hospitalité des humains envers les jnûn, que s'expriment et se maintiennent les liens entre les humains et les non-humains, les vivants et les morts. Les actions humaines dont nous avons parlé sont marquées par des transferts alimentaires, et elles placent en évidence un ensemble d'échanges mettant en jeu un système de dons et de contre-dons. La commensalité rituelle semble propice à articuler un type de relations entre des sphères ontologiques séparées. Je n'ai pu qu'esquisser ici certains contours de notre sujet, certains traits communs qui donnent lieu à l'établissement d'une problématique précise. Il faudrait se plonger dans le détail des rituels, afin de reconnaître les logiques interactives de l'inclusion et de l'exclusion du monde des humains et du monde des non-humains qui configurent la définition des ontologies locales. Une tâche qui passe par l'ethnographie et le travail de terrain. 


\section{BIBLIOGRAPHIE}

Amster E. J. 2013 - Medicine and the saints: Science, Islam and the Colonial Encounter. Texas, University of Texas Press.

Antonioli M. 2014 - Théories et pratiques écologies : de l'écologie urbaine à l'imagination environnementale. Paris, Presses Universitaires de Paris-Ouest.

Aufauvre C. 2009 - Des procès en chair et en songe. Sainteté et exorcisme à Bouya Omar et Sidi Chamharouch (Maroc). Altérités 6 (2) : 93-114.

Benkheira H. 1999 - Le rite à la lettre. Régime carné et normes religieuses. In : Bonte P., Brisebarre A.-M. \& Gokalp A. (Ed.) Sacrifices en islam. Espaces et temps d'un rituel. Paris, CNRS Éditions : 63-91.

Berger H. 1992 - Contes du Maroc. Paris, Gründ.

Bloch M. 2005 - Commensality and poisoning. Essays on cultural transmission. Oxford, Berg.

Bonte P. 1999 - Sacrifices en Islam. Textes et contextes. In : Bonte P., Brisebarre A.-M. \& Gokalp A. (Ed.) Sacrifices en islam. Espaces et temps d'un rituel. Paris, CNRS Éditions : 21-61.

Boudou B. 2012 - Éléments pour une anthropologie politique de l'hospitalité. Revue du MAUSS 40 (2) : 267-284.

Candea M. 2012 - Derrida en Corse? Hospitality as scale-free abstraction. Journal of the Royal Anthropological Institute 18 (s1) : S34-S48.

Candea M. \& Da Col G. 2012 - The return to hospitality. Journal of the Royal Anthropological Institute 18 (s1) : S1-S19.

Camps-Fabrer H. 2000 - Hospitalité. Encylopédie berbère 23. Hiempsal-Icosium, Aix-en-Provence, Édisud : 3503-3508.

Cixous H. 2003 - Le dernier caravansérail (programme). Paris, Le Théâtre du Soleil.

Crapanzano V. 1973 - The Hamadscha, A study in Moroccan ethnopsychiatry. Berkeley, University of California Press.

Da Col G. 2014 - Turns and returns. HAU, Journal of Ethnographic Theory 4 (1) : i-v.

Derrida J. 1998 - Un ver à soie. In : Cixous H. \& Derrida J. (Ed.) Voiles. Paris, Galilée : 23-85.

Derrida J. \& Dufourmantelle A. 1997 - De l'hospitaité. Anne Dufourmantelle invite Jacques Derrida à répondre. Paris, Calmann-Lévy.

Descola P. 1993 - Les affinités sélectives. Alliance, guerre et prédation dans l'ensemble jivaro. L'homme 33 (2-4) : 171-190.

Descola P. 2005 - Par-delà nature et culture. Paris, Gallimard.

El Alaoui N. 2001 - Le Soleil, la Lune et la Fiancée végétale : essai d'anthropologie rituelle. Les Idaw Martini de l'Anti-Atlas. Aix-en-Provence, Edisud.

Epton N.C. 1958 - Saints and sorcerers: A Moroccan journey. London, Cassell.

Erb M. 2013 - Gifts from the Other Side: Thresholds of Hospitality and Morality in an Eastern Indonesian Town. Oceania 83 (3) : 295-315. 
Farès B. 1932 - L'honneur chez les Arabes avant l'Islam : étude de sociologie. Paris, AdrienMaisonneuve.

Garrigues-Cresswell M. 1994 - La profusion rituelle berbère : une nécessité sociale. Annuaire de l'Afrique du Nord XXXIIII : 181-188.

Gélard M.-L. (Ed.) 2008 - Les usages du henné : pratiques, rites et représentations symboliques. Nancy, Presses Universitaires de Nancy.

Gélard M.-L. 2010 - « Mon sel dans ton ventre ». Accord, agrégation et identité par la consommation de nourriture carnée dans le sud-est marocain. Anthropozoologica 45 (1) : 67-76.

González Vázquez A. 2010 - Mujeres de Yebala: Género, Islam y alteridades en Marruecos. Thèse doctorale, Universidad de Cantabria.

González Vázquez A. 2013 - Usos de la terminología del parentesco en Marruecos: Humanos, jnun, matrimonio, afinidad y alianza. Ankulegi, Revista de Antropología Social 17 : 57-70.

Gotman A. 2001 - Le sens de l'hospitalité. Essai sur les fondements sociaux de l'accueil de l'autre. Paris, PUF.

Guattari F. 1989 - Les trois écologies. Paris, Éditions Galilée.

Hell B. 2002 - Le tourbillon des génies. Au Maroc avec les Gnaoua. Paris, Flammarion.

Henare A., Holbraad M. \& Wastell S. 2007 - Thinking through things. Theorising artefacts ethnographically. London, Routledge.

Herzfeld M. 1980 - Honor and Shame. Problems in the Comparative Analysis of Moral Systems. Man 15 : 339-351.

Herzfeld M. 1987 - « As in your house »: Hospitality, Ethnography, and the Stereotypes of Mediterranean Society. In : Gilmore D.D. (Ed.) Honour and Shame and the Unity of the Mediterranean. Washington, AAA : 75-89.

Herzfeld M. 2008 - L'intimité culturelle. Poétique sociale dans l'État nation. Laval, Presses de l'Université de Laval, 285 p.

Holbraad M. \& Pedersen M.A. 2017 - The Ontological turn. An Anthropological exposition. Cambridge, Cambridge University Press.

Houdart S. \& Thiery O. 2011 - Humains, Non-Humains. Comment repeupler les Sciences Sociales. Paris, La Découverte.

Innerarity D. 2009 - Éthique de l'hospitalité. Québec, Presses de l'Université Laval.

Kant I. 1795 - Perpetual Peace: A philosophical sketch.

Kapchan D. 2007 - Traveling Spirit Masters: Moroccan Gnawa Trance and Music. Wesleyan University Press.

Kirksey S.E. \& Helmreich S. 2010 - The emergence of multispecies ethnography. Cultural anthropology 25 (4) : 545-576.

Kohn E. 2007 - How dogs dream: Amazonian natures and the politics of transspecies engagement. American ethnologist 34 (1) : 3-24.

Ladwig P. 2011 - Can things reach the dead? The ontological status of objects and the study of Lao budhist rituals for the spirits of the deceased. In : Endres K.W. \& Lauser A. (Ed.) Engaging the Spirit World: Popular Beliefs and Practices in Modern Southeast Asia. London, Berghahn : 19-41. 
Ladwig P. 2012a - Visitors from hell: transformative hospitality to ghosts in a Lao Buddhist festival. Journal of the Royal Anthropological Institute 18 (s1) : S90-S102.

Ladwig P. 2012b - Ontology, materiality and spectral traces: Methodological thoughts on studying Lao Buddhist festivals for ghosts and ancestral spirits. Anthropological Theory 12 (4) : 427-447.

Lambek M. 1996 - Afterword: spirits and their stories. In : Mageo J.M., Howard A. \& Levy R. (Ed.) Spirits in Culture, History and Mind. London, Routledge : 237-250.

Legey F. 2010 (1926) - Contes et légendes populaires du Maroc recueillis à Marrakech. Casablanca, Éditions du Sirocco.

Lens A.R. de 1925 - Pratiques des harems marocains : Sorcellerie, médicine, beauté. Paris, Geuthner.

Maarouf M. 2009 - 'Āšūrā' as a Female Ritual Challenge to Masculinity. Arabica 56 (4-5) : 400-439.

Mahdi M. 1999 - Pasteurs de l'Atlas. Production pastorale, droit et rituel. Casablanca, Fondation Konrad Adenauer.

Mallet M.-L. 2008 - Une pensée de l'hospitalité. In : Derrida à Alger : Un regard sur le monde. Paris/ Alger, Actes Sud/Barzakh.

Massignon L. 1987 - L'hospitalité sacrée. Paris, Nouvelle Cité.

Mateo Dieste J.L. 2014 - ¿Eres musulmán, judío o cristiano? Alteridad y construcción de la diferencia en el exorcismo y el adorcismo marroquíes. Revista de Dialectología y tradiciones populares LXIX (2) : 263-284.

Morgan L.H. 1881 - Houses and House-Life of the American Aborigines. Washington, Government Printing Office.

Naess A. 1973 - The shallow and the deep, long-range ecology movement. A summary. Inquiry, An interdisciplinary journal of philosophy 16 (1-4) : 95-100.

Pandolfo S. 1997 - Impasse of the Angels: Scenes from a Moroccan Space of Memory. Chicago and London, University of Chicago Press.

Pitt-Rivers J. 1968 - The Stranger, the Guest and the Hostile Host. In : Peristiany J.G. (Ed.)

Contributions to Mediterranean Sociology. The Hague, Mouton : 13-30.

Pitt-Rivers J. 1977 - The law of hospitality. In : Pitt-Rivers J. (Ed.) The fate of Shechem, or the politics of sex : essays in the anthropology of the Mediterranean. Cambridge, University Press : 94-112.

Pitt-Rivers J. 2012 - The law of hospitality. HAU, Journal of Ethnographic Theory 2 (1) : 501-517.

Rachik H. 1990 - Sacré et sacrifice dans le Haut-Atlas marocain. Casablanca, Afrique Orient.

Rhani Z. 2007 - Possession et exorcisme au Maroc : Les rituels de l'hospitalité. In : Strohm K. \&

Lanoue G. (Ed.) Célébrer une vie. Actes du colloque en l'honneur de Jean-Claude Muller. Montréal, Publication de l'Université de Montréal : 98-106.

Rhani Z. 2008 - Le cherif justicier et la possédée nourricière : Les rituels de l'hospitalité, chapitre dans la thèse doctorale intitulée Le culte de Ben Yeffou : sainteté, rituel et pouvoir au Maroc. Faculté des Arts et des Sciences, Université de Montréal.

Rivers J. 1977 - The law of hospitality. In : Pitt-Rivers J. (Ed.) The fate of Schechem or the Politics of Sex: Essays in the Anthropology of the Mediterranean. Cambridge, Cambridge University Press : 94-112.

Skounti A. 2006 - «L'hospitalité berbère » prolongements d'un récit gellnerien. Études et Documents Berbères 24 : 149-158. 
Shérer R. 1997 - Cosmopolitisme et hospitalité. Communications 65 (1) : 59-68.

Simenel R. 2012 - Quand les djinns sèment le doute dans l'ordre des apparences : un contrepoint animiste dans l'ontologie analogique marocaine. In : Ce qui fait les êtres : la caractérisation des êtres naturels et surnaturels. Les rencontres du Centre Jacques Berque, Rabat : 13-19.

Spadola E. 2013 - The Calls of Islam. Sufis, Islamists and Mass mediation in Urban Morocco. Bloomington, Indiana University Press.

Viveiros de Castro E. 1998 - Les pronoms cosmologiques et le perspectivisme amérindien. In : Alliez E. (Ed.) Gilles Deleuze. Une vie philosophique. Le Plessis-Robinson, Institut Synthélabo : 429-462.

Viveiros de Castro E. 2002 - A inconstância da alma selvagem. E outros ensaios de antropología. São Paulo, Cosac Naify.

Viveiros de Castro E. 2009 - Métaphysiques cannibales. Paris, Presses Universitaires de France.

Wagner R. 2012 - « Luck in the double focus »: ritualized hospitality in Melanesia. Journal of the Royal Anthropological Institute 18 (s1) : S161-S174.

Walentowitz S. 2006 - Tout un monde au creux d'un plat. Propos sur la grande écuelle des Touaregs. Journal des africanistes 76 (1) : 103-120.

Westermarck E. 1926 - Ritual and belief in Morocco. London, Macmillan and Co.

\section{NOTES}

1. Voir aussi les articles de Catherine Allerton, Caroline Humphrey, Patrice Ladwig, Grégory Delaplace, Roy Wagner, Carlos Fausto et al. dans le volume 18 du Journal of the Royal Anthropological Institute, edité par Matei Candea et Giovanni Da Col en 2012, intitulé « The return to hospitality: strangers, guests, and ambiguous encounters ». Voir aussi les articles : Erb, 2013 ,Ladwig 2012a, Ladwig 2012 b et Ladwig 2011.

2. Ce mot est souvent traduit comme «tribu » ou « lignage ».

3. Suite à mon travail de thèse, en 2011 et 2012, j'ai développé un projet postdoctoral intitulé Soufisme, corporéités et altérités au Maroc: corps féminin et corps masculin dans la confrérie Baqqaliyya, au Laboratoire d'Anthropologie Sociale du Collège de France, encadré par Tassadit Yacine, continué en 2013 à l'Université du Pays-Basque, financé par le Gouvernement Basque.

4. Dans la thèse de Zakaria Rhani, le chapitre est intitulé «Le cherif justicier et la possédée nourricière : Les rituels de l'hospitalité » (Rhani 2008 : 261-262).

\section{RÉSUMÉS}

L'hospitalité, plus particulièrement son caractère normatif, est un des thèmes classiques de l'anthropologie sociale, souvent traité à propos des sociétés méditerranéennes. En dépit d'un regain d'intérêt récent de l'anthropologie sociale pour le thème de l'hospitalité, l'imaginaire et la pratique de l'hospitalité interspécifique demeurent peu explorés, en particulier ce qu'ils nous disent, par exemple, sur les conceptions de l'altérité, de l'humanité et de l'animalité, sur les 
ontologies locales, sur le raisonnement éthique, sur l'activité rituelle des femmes et des hommes, et sur les relations entre les êtres vivants, notamment sur les relations entre les êtres humains et les êtres non-humains. Notre article s'inscrit dans cette ligne de réflexion sur l'Autre, et s'attache particulièrement à explorer les enjeux de l'hospitalité interspécifique au nord du Maroc.

Hospitality, in particular its normative character, is one of the classical themes of social anthropology, often dealt with in relation to Mediterranean societies. Despite a renewed interest for the theme of hospitality in social anthropology, both the imaginary and the practice of interspecific hospitality remain little explored, notably what they tell us, for example, on the conceptions of otherness, humanity and animality, on local ontologies, on ethical reasoning, on the ritual activity of women and men, and on the relations between living beings, particularly on the relationship between human beings and non-human beings. Our article is part of this line of reflection on the Other, and is especially interested in exploring the stakes of interspecific hospitality in Northern Morocco.

INDEX

Keywords : hospitality, non-human beings, jnûn, Jbala, Ghomara, Morocco

Mots-clés : hospitalité, non-humains, jnûn, Jbala, Ghomara, Maroc

\section{AUTEUR}

ARACELI GONZÁLEZ VÁZQUEZ

Cientifica titular (Chercheuse titulaire)

Institución Milà i Fontanals (IMF), CSIC - Carrer de les Egipcíaques, 15 - 08001 Barcelona 